\title{
EXPERIMENTAL OBSERVATIONS OF THE LASER KEYHOLE WELDING PROCESS OF AA5182
}

\author{
B.J. Aalderink ${ }^{1}$, R.G.K.M. Aarts ${ }^{2}$, J.B. Jonker ${ }^{2}$ and J. Meijer ${ }^{2}$ \\ ${ }^{1}$ Netherlands Institute for Metals Research \\ P.O. Box 217, 7500 AE Enschede, The Netherlands \\ 2 University of Twente, Laboratory of Mechanical Automation \\ P.O. Box 217, 7500 AE Enschede, The Netherlands
}

b.j.aalderink@nimr.nl

\begin{abstract}
Monitoring systems for the laser keyhole welding process of aluminium Tailor Welded Blanks, are in many cases vital to ensure a certain weld quality. Especially process visualization with camera based systems gives a lot of insight. Although for steel it has already been demonstrated that images can be obtained in real-time from the laser welding process using these cameras based on silicon chips, for aluminium this turns out to be not so easy. The light emitted by the weld plume hides the weld pool from the coaxially mounted camera.

In this paper recent experiments are discussed in which a monitoring system is used, that is composed of low cost standard components, to visualize the CW Nd:YAG laser keyhole welding process of AA5182. This monitoring system utilizes a diode laser to illuminate the welding process, combined with an optical interference filter and a CMOS camera. In this way the monitoring system is not overradiated by the optical emissions of the sample material. It proved to be possible to eliminate the influence of the light emitted by the weld plume on the image and to detect the melt pool. Future efforts will focus on visualizing the keyhole in these images.
\end{abstract}

\section{Introduction}

The industrial demand for first-time-right high quality joints drives the development of spatially resolving monitoring and control systems for the laser welding process. In the past there have been many attempts to develop such a system, using different types of cameras and optical filtering techniques. These monitoring systems usually record the light emitted by the welding process and relate this to specific welding defects (e.g. $[5,3,2])$. However es- pecially for the laser keyhole welding process of aluminium this proofs to be difficult. Only with high end equipment like high speed or infrared cameras it was possible to visualize the melt pool and keyhole during welding $[3,4]$. However this way of weld pool visualization has some serious drawbacks.

The most important drawback is that the optical emissions of the welding process are very process dependant. This means that a camera based monitoring system based on these emissions, that works well for one set of materials and parameters, will have a much lower performance for another set.

Additionally both high speed cameras and infrared cameras are very expensive compared to conventional silicon based camera systems. Also high speed cameras have the disadvantage that they are very bulky, preventing a compact monitoring solution and that it is not always possible to incorporate high speed recordings in a closed loop system.

In this paper some first experimental results are described of a monitoring system that was developed to visualize the $\mathrm{CW}$ Nd:YAG laser welding process of AA5182-H111 Tailor Welded Blanks (TWB's). This system utilizes an external illumination source in combination with a low cost Si based CMOS camera mounted in a coaxial setup. Since the camera image is formed by the light from the external illumination source, it is expected that this monitoring system can also be used to monitor the laser welding process of other materials. The light emitted by the welding process only degrades the image quality. This is expressed in the Signal-to-Noise $(S / N)$ ratio that is defined as:

$$
S / N=\frac{I_{S}}{I_{N}},
$$

where $I_{S}$ is the irradiance of the reflected illumination light that reaches the camera chip and $I_{N}$ is 


\begin{tabular}{|l|c|}
\hline Element & Content [mass\%] \\
\hline \hline $\mathrm{Mg}$ & 4.90 \\
\hline $\mathrm{Mn}$ & 0.26 \\
\hline $\mathrm{Cu}$ & 0.11 \\
\hline $\mathrm{Si}$ & 0.08 \\
\hline $\mathrm{Ti}$ & 0.01 \\
\hline $\mathrm{Al}$ & Bal. \\
\hline
\end{tabular}

Table 1: The amount of alloying elements present in AA5182-H111 (information provided by Corus).

\begin{tabular}{|l|c|}
\hline Seam configuration & Bead-on-plate \\
\hline Plate thickness & $1.1 \mathrm{~mm}$ \\
\hline Laser light wavelength & $1064 \mathrm{~nm}$ \\
\hline Laser power & $3000 \mathrm{~W}(\mathrm{CW})$ \\
\hline Welding speed & $100 \mathrm{~mm} / \mathrm{s}$ \\
\hline Shielding gas & $\mathrm{Ar}$ \\
\hline Shielding gas top flow & $1360 \mathrm{l} / \mathrm{h}$ \\
\hline Shielding gas backing flow & $340 \mathrm{l} / \mathrm{h}$ \\
\hline Focal distance & $150 \mathrm{~mm}$ \\
\hline Focal diameter $^{a}$ & $450 \mu \mathrm{m}$ \\
\hline Focal position & $0 \mathrm{~mm}$ \\
\hline
\end{tabular}

Table 2: The most important parameter values used during the measurements

${ }^{a}$ Relative to top surface of the sample

matches the wavelength of the diode laser light, so only this light is focussed on the camera chip.

The used camera system was a CCAM $\mathrm{CCf}_{1000}$ system, which contains a silicon based Fuga 1000 CMOS chip. A CMOS chip is especially advantageous for visualization of sceneries containing large intensity differences, due to its large dynamical range and the ability to define a specific RegionOf-Interest (ROI) on the chip.

To obtain usable geometrical information about the weld pool during welding, the $\mathrm{S} / \mathrm{N}$ ratio has to be sufficiently large. The minimal value will be denoted with $S / N_{\min }$ and will be about one. Several strategies can be followed to raise $S / N$ above its critical value.

Without optical filtering $I_{S}$ and $I_{N}$ are defined as:

$$
\begin{aligned}
& I_{S}=\int I_{\lambda S} d \lambda \\
& I_{N}=\int I_{\lambda N} d \lambda .
\end{aligned}
$$

Here $I_{\lambda S}$ and $I_{\lambda N}$ are respectively the irradiance of the reflected illumination light that reaches the camera chip per unit wavelength and the irradiance of the welding process itself that reaches the camera chip per unit wavelength. Since $I_{N}$ is determined by the emissions of the welding process and cannot easily be influenced independently from $I_{S}$, the only way to obtain a sufficiently large $\mathrm{S} / \mathrm{N}$ ratio is to have an sufficiently strong illumination source to assure that $I_{S} \geq\left(S / N_{\min } \cdot I_{N}\right)$. Empirically it has been found that $I_{N}$ is quite large, which would result in the necessity of a bulky illumination solution, which is undesirable. 


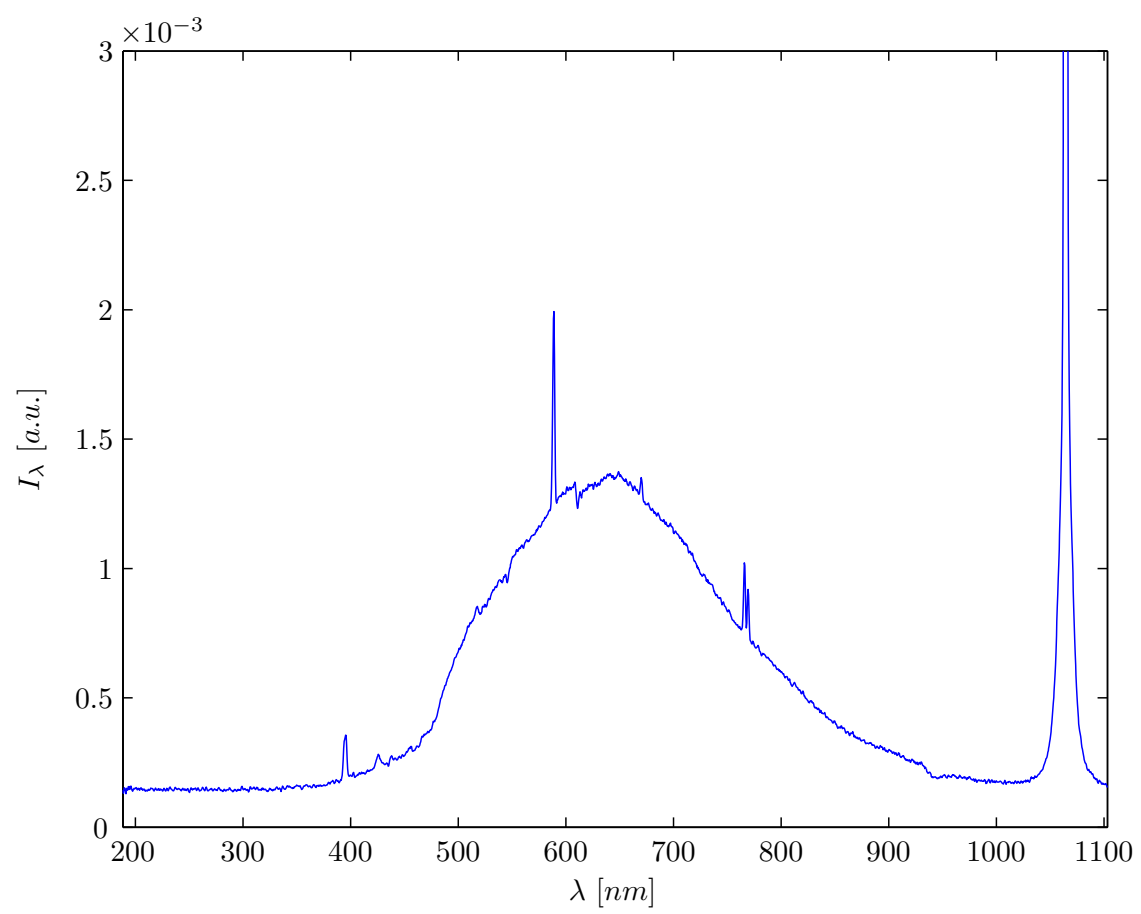

Figure 1: The measured uncalibrated emission spectrum during the Nd:YAG laser welding of AA5182-H111. The vertical axis contains the irradiance per unit wavelength range. On the horizontal axis the wavelength is given.

In this case it was chosen to use a bandpass filter in combination with a matching illumination source. Here $I_{S}$ and $I_{N}$ can be defined as follows:

$$
\begin{aligned}
I_{S} & =\int T_{\lambda} I_{\lambda S} d \lambda \\
I_{N} & =\int T_{\lambda} I_{\lambda N} d \lambda .
\end{aligned}
$$

where $T_{\lambda}$ is the transmittance of the optical filter per unit wavelength. The optical filter has a near gaussian shaped transfer function with a peak transmission of $65 \%$ around $810 \mathrm{~nm}$ and a FWHM of 10 $\mathrm{nm}$. This means that $I_{N}$ is reduced to only a few percent of its original value, since only process light in a very small wavelength band can reach the camera chip. For illumination a $1.8 \mathrm{~W}$ diode laser source with a wavelength of $810 \mathrm{~nm}$ was used. This wavelength was chosen since the process emissions are not very strong in this spectral region (no peaks in the spectrum) and since it is possible to find reasonably priced high power diode lasers at this wavelength. Since the laser light has a very narrow wavelength band, which lies well within the FWHM of the optical filter, $I_{S}$ is only reduced by approximately 30 to $40 \%$. This means that the $\mathrm{S} / \mathrm{N}$ ratio will increase with approximately two orders of magnitude. The diode laser was used to illuminate a sample area of $3.0 \times 4.2 \mathrm{~mm}$. This results in an irradiance of $1.4 \cdot 10^{5}$ $\mathrm{W} / \mathrm{m}^{2}$ at the welding process.

\section{Laser Welding System and Parameters}

To test the monitoring system welding experiments were carried out using $1.1 \mathrm{~mm}$ AA5182-H111 sheets. In table 1 the weight percentage of the alloying elements of this material are given. During these experiments a $4 \mathrm{~kW}$ Trumpf THL4006D CW Nd:YAG laser source was used in combination with a $600 \mu \mathrm{m}$ core optical fiber and a standard Trumpf welding head. The sample was moved under the laser spot using a XY manipulator, controlled by a DMC1000 Galil motion controller. The process was shielded from the environment by shielding gas both from the top, by means of a gas nozzle on the welding head, as from the bottom, by a gas supply in the sample holder.

In table 2 an overview of the most important welding parameters is given. These parameters resulted in a keyhole welding mode that completely penetrated the material. 


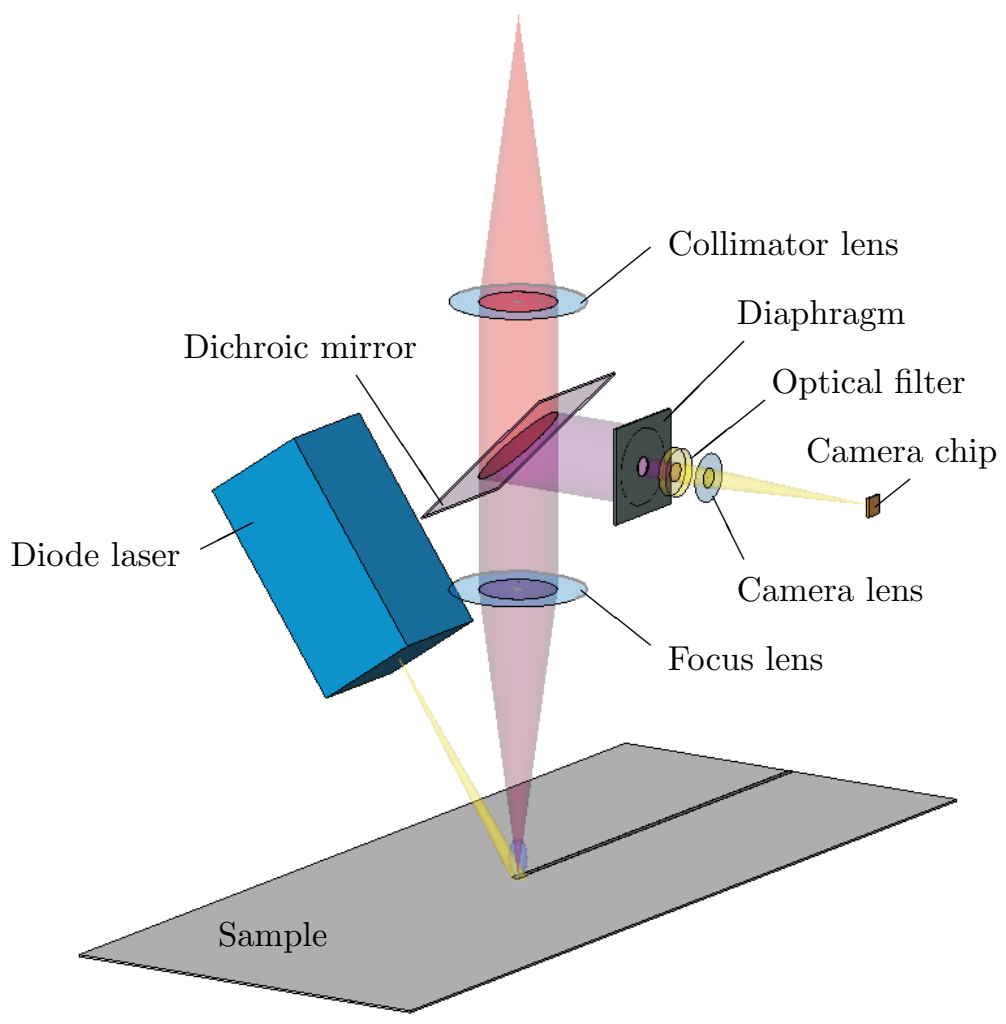

Figure 2: A schematic overview of the coaxial monitoring system with external illumination.

\section{Results and Discussion}

Experiments were carried out using the aforementioned laser welding and monitoring setup. For reference these experiments were also conducted without external illumination but with the optical filter in the optical path. In figure 3 and 4 typical camera images for these two situations are displayed. Note that the contrast in both figures has been optimized.

In the situation without external illumination only light originating from the process itself reaches the camera chip. Although there is a bright spot visible at the place of the laser spot, no clear keyhole or melt contours are visible. Similar results were obtained using interference filters with different central wavelengths. If however the external illumination is switched on, the camera image changes dramatically, as can be seen in figure 4. Centrally in the image a pattern of bright spots can be detected, which is highly dynamic. In figure 5 this region has been highlighted. This pattern can be explained by diode laser light reflecting of the billowing melt surface. The bright spots are somewhat smeared out in the welding direction due to the shutter effect, caused by the serial readout of the camera chip.

Around this melt a darker contour is visible (see figure 6). The dimensions of this contour have been compared to the weld bead after welding, resulting in a perfect match. These aspects indicate that the darker contour corresponds to the melting front.

In figure 4 it is however not yet possible to distinguish a keyhole. A possible cause is the high frequency oscillations of the keyhole walls compared to the frame rate of the camera. Also the lack of sharpness degrades the image quality. This might be caused by the particles of the weld plume in the optical path of the camera and fluctuations in the refraction index in the weld plume.

\section{Conclusions}

The main conclusions that can be drawn are:

1. The described monitoring system is able to visualize the melting front during the laser welding of AA5182, using standard components.

2. Since this system does not heavily depend on the optical emissions of a specific welding process, it is expected that this system can also be used for monitoring of the laser welding of materials other than aluminium. 


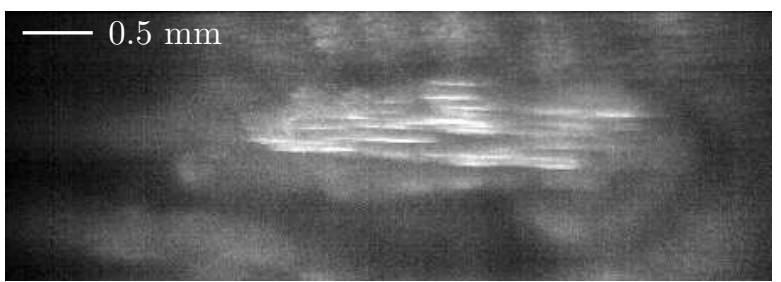

Figure 3: A typical CMOS camera image with $810 \mathrm{~nm}$ optical interference filter, without external illumination. The contrast of the image has been optimized.

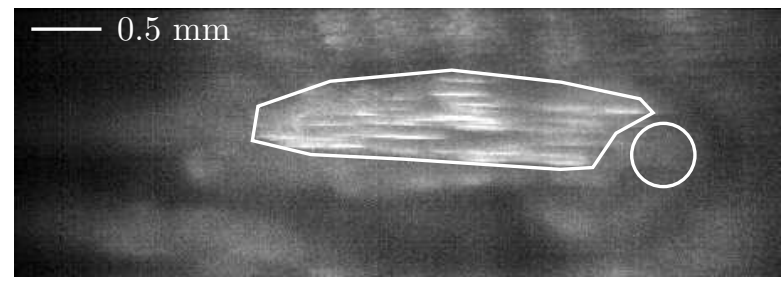

Figure 4: A typical CMOS camera image with $810 \mathrm{~nm}$ optical interference filter, with external illumination. The contrast of the image has been optimized.

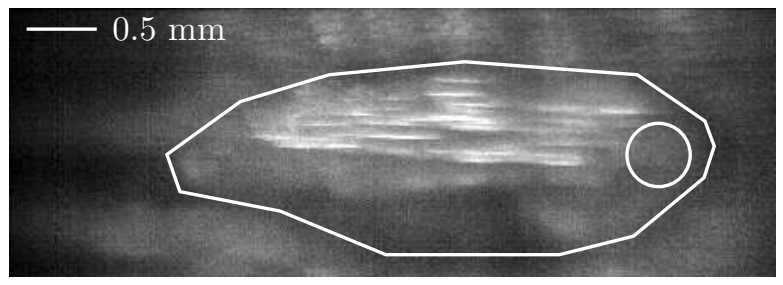

Figure 5: Indication of the area where the pattern of bright spots is visible in the CMOS camera image. Also the laser spot is indicated in this picture.

3. It is not yet possible to distinguish the keyhole during the laser welding process of AA5182 using this system.

Further research will focus on the optimization of the image quality and the process illumination. A following step is to link specific welding defects to features in the camera images and to automatically detect these features.

\section{Acknowledgements}

This research was carried out under the project number MC8.02116 in the framework of the Strategic Research Program of the Netherlands Institute of Metals Research (www.nimr.nl). The authors wish to thank Corus for their continuing support.

\section{References}

[1] B.J. Aalderink, R.G.K.M. Aarts, J.B. Jonker, and J. Meijer. Weld Plume Emissions During Nd:YAG Laser Welding. Proceedings of LIM '05, 2005.

[2] J. Beersiek. On-line monitoring of Keyhole Instabilities during Laser Beam Welding. Proceedings of ICALEO '99, pages D49 - D58, 1999.

[3] R.E. Müller, H. Gu, N. Ferguson, M. Ogmen, and W.W. Duley. Real Time Optical Spectral

Figure 6: Indication of the dark contour corresponding to the melt front in the CMOS camera image. Also the laser spot is indicated in this picture.

Monitoring of Laser Welding Plumes. Proceedings of ICALEO '98, pages C132 - C138, 1998.

[4] J. Müller-Borhanian, C. Deininger, F.H. Dausinger, and H. Hügel. Spatially Resolved On-Line Monitoring During Laser Beam Welding of Steel and Aluminum. Proceedings of ICALEO '04, 2004.

[5] C. Peters, M.D.T. Fox, F.M. Haran, D.P. Hand, J.D.C. Jones, and W.M. Steen. Nd:YAG Welding Penetration-Monitoring using BackScattered Laser Light from in and around the Keyhole. Proceedings of ICALEO '98, pages C149 - C157, 1998.

\section{Meet the Author}

B.J. Aalderink is a $\mathrm{PhD}$ researcher working for the NIMR. He holds an M.Sc in Applied Physics and his main interests lie in the field of system and control engineering, robotics and laser welding technology. 